\title{
A Four-Order Linear Recurrence Formula Involving the Quartic Gauss Sums and One Kind Two-Term Exponential Sums
}

\author{
Lan Qi ${ }^{1}$ and Xingxing $\operatorname{Lv} \mathbb{D i D}^{2}$ \\ ${ }^{1}$ School of Mathematics and Statistics, Yulin University, Yulin, Shaanxi, China \\ ${ }^{2}$ School of Mathematics, Northwest University, Xi'an, Shaanxi, China \\ Correspondence should be addressed to Xingxing Lv; lvxingxing@stumail.nwu.edu.cn
}

Received 24 February 2021; Revised 9 March 2021; Accepted 22 March 2021; Published 2 April 2021

Academic Editor: Tianping Zhang

Copyright ( 2021 Lan Qi and Xingxing Lv. This is an open access article distributed under the Creative Commons Attribution License, which permits unrestricted use, distribution, and reproduction in any medium, provided the original work is properly cited.

The main purpose of this paper is using the analytic method and the properties of the classical Gauss sums to study the computational problem of one kind hybrid power mean involving the quartic Gauss sums and two-term exponential sums and give an interesting four-order linear recurrence formula for it. As an application, we can obtain all values of this kind hybrid power mean with mathematica software.

\section{Introduction}

In the number theory textbooks, especially the elementary number theory and the analytic number theory textbooks, there are many contents related to primes, such as famous prime number theorem and Dirichlet's theorem. However, maybe the most important and most profound property related to primes is that any prime $p$ with $p=4 k+1 \mathrm{can}$ be expressed as the square sums of two positive integers. That is, $p=\alpha^{2}+\beta^{2}$. And, more precisely (see Theorems 4-11 of [1]),

$$
p=\alpha^{2}+\beta^{2} \equiv\left(\sum_{a=1}^{(p-1 / 2)}\left(\frac{a+r \bar{a}}{p}\right)\right)^{2}+\left(\sum_{a=1}^{(p-1 / 2)}\left(\frac{a+n \bar{a}}{p}\right)\right)^{2}
$$

where $(* / p)$ denotes Legendre's symbol modulo $p, r$ and $n$ are any two integers such that $(r n / p)=-1$, and $\bar{a}$ satisfies the congruence equation $a \cdot \bar{a} \equiv 1 \bmod p$.

This conclusion has been extended by Professor W. P. Zhang in an unpublished paper. That is, he proved the following two conclusions. Let $p$ be an odd prime with $p \equiv 1 \bmod 4$. Then, for any nonprincipal even character $\chi(\bmod p)$, one has the identity

$$
p=\left|\sum_{a=1}^{(p-1 / 2)} \chi(a+r \bar{a})\right|^{2}+\left|\sum_{a=1}^{(p-1 / 2)} \chi(a+n \bar{a})\right|^{2},
$$

where $r$ and $n$ are any two integers such that $(r n / p)=-1$.

Let $p$ be an odd prime with $p \equiv 7 \bmod 12$. Then, for any nonreal character $\chi \bmod p$ with $\chi^{3} \neq \chi_{0}$, the principal character $\bmod p$, one has

$$
\begin{aligned}
p= & \left|\sum_{a=1}^{(p-1 / 3)} \chi^{3}\left(a^{2}+\bar{a}\right)\right|^{2}+\left|\sum_{a=1}^{(p-1 / 3)} \chi^{3}\left(a^{2}+t \bar{a}\right)\right|^{2} \\
& +\left.|| \sum_{a=1}^{(p-1 / 3)} \chi^{3}\left(a^{2}+t^{2} \bar{a}\right)\right|^{2},
\end{aligned}
$$

where $t$ is any integer such that $p \mid t^{3}-1$ and $p \nmid t-1$.

Unfortunately, formulas (1) and (2) are only suitable for the primes $p$ with $p=4 k+1$. If $p=4 k+3$, we still have not found any similar representation. Some related works can also be found in [2-12].

On the other hand, in $[10,11], \mathrm{X} . \mathrm{X} \mathrm{Li}$ and S. M. Shen studied the mean value properties of the quartic Gauss sums and two-term exponential sums and proved an interesting recurrence formula for it. That is, they give a recurrence formula for 


$$
M_{k}(p)=\sum_{m=1}^{p-1}\left(\sum_{b=0}^{p-1} e\left(\frac{m b^{4}}{p}\right)\right)^{k} \cdot\left(\sum_{a=1}^{p-1} e\left(\frac{m a^{4}+a}{p}\right)\right)^{h}
$$

with $h=2$ and 3 , respectively.

Inspired by literatures $[10,11]$, we considered the calculating problem of the hybrid power mean

$$
H_{k}(p)=\sum_{m=1}^{p-1}\left(\sum_{b=0}^{p-1} e\left(\frac{m b^{4}}{p}\right)\right)^{k} \cdot\left|\sum_{a=1}^{p-1} e\left(\frac{m a^{4}+a}{p}\right)\right|^{2}
$$

Of course, the contents in [10] and $H_{k}(p)$ look a little similar, but they are very different. The main difference between them is the absolute value of the two-term exponential sums. In fact, for all positive integers $k \geq 1$, we have

$$
\left|\sum_{a=1}^{p-1} e\left(\frac{m a^{4}+a}{p}\right)\right|^{k} \neq\left(\sum_{a=1}^{p-1} e\left(\frac{m a^{4}+a}{p}\right)\right)^{k}
$$

Our goal is to obtain a sharp asymptotic formula for $H_{k}(p)$. About this problem, it seems that none had studied it yet, at least we have not seen related papers before. The problem is interesting because it may reveal the regularity of the distribution of values related to the quartic Gauss sums and two-term exponential sums.

In this paper, we will use the analytic methods and the properties of the classical Gauss sums to study this problem and prove an interesting four-order linear recurrence formula for it. That is, we will prove the following.

Theorem 1. Let $p$ be an odd prime with $p \equiv 1 \bmod 8$. Then, for any integer $k \geq 1$, we have the four-order linear recurrence formula:

$$
H_{k+4}(p)=6 p H_{k+2}(p)+8 p \alpha H_{k+1}(p)-\left(p^{2}-4 p \alpha^{2}\right) H_{k}(p),
$$

where the first four terms of $H_{k}(p)$ are $H_{1}(p)=6 p \cdot(1-\alpha)$,

$$
\begin{aligned}
H_{2}(p)= & 3 p^{3}-20 p^{2}-4 p \alpha^{2}+12 p \alpha-3 p \\
H_{3}(p)= & 6 p^{3} \alpha-66 p^{2} \alpha-6 p \alpha+8 p \alpha^{2}+34 p^{2}, \\
H_{4}(p)= & 17 p^{4}+4 p^{3} \alpha^{2}-88 p^{2} \alpha^{2}-116 p^{3} \\
& -17 p^{2}+120 p^{2} \alpha-4 p \alpha^{2} .
\end{aligned}
$$

Theorem 2. Let $p$ be an odd prime with $p \equiv 5 \bmod 8$. Then, for any integer $k \geq 1$, we have the recurrence formula

$$
H_{k+4}(p)=-2 p H_{k+2}(p)+8 p \alpha H_{k+1}(p)-\left(9 p^{2}-4 p \alpha^{2}\right) H_{k}(p)
$$

where the first four terms of $H_{k}(p)$ are $H_{1}(p)=2 p \cdot(\alpha+1)$,

$$
\begin{aligned}
& H_{2}(p)=-p^{3}+4 p^{2}+p+4 p \alpha^{2}+4 p \alpha, \\
& H_{3}(p)=-10 p^{2}+2 \alpha \cdot\left(3 p^{3}-17 p^{2}-3 p\right), \\
& H_{4}(p)=4 p^{3} \alpha^{2}-7 p^{4}-8 p^{2} \alpha^{2}+28 p^{3}-4 p \alpha^{2}+7 p^{2}+8 p^{2} \alpha .
\end{aligned}
$$

If $p \equiv 1 \bmod 8$, then there exists an integer $c$ such that $c^{4} \equiv-1 \bmod p$. So, from Theorem 1 , we may immediately deduce the following two corollaries.

Corollary 1. Let $p$ be an odd prime with $p \equiv 1 \bmod 8$. Then, we have

$$
\sum_{m=1}^{p-1}\left|\sum_{b=0}^{p-1} e\left(\frac{m b^{4}}{p}\right)\right|^{2} \cdot\left|\sum_{a=1}^{p-1} e\left(\frac{m a^{4}+a}{p}\right)\right|^{2}=3 p^{3}-20 p^{2}-4 p \alpha^{2}+12 p \alpha-3 p
$$

Corollary 2. Let $p$ be an odd prime with $p \equiv 1 \bmod 8$. Then,

we have

$$
\sum_{m=1}^{p-1}\left|\sum_{b=0}^{p-1} e\left(\frac{m b^{4}}{p}\right)\right|^{4} \cdot\left|\sum_{a=1}^{p-1} e\left(\frac{m a^{4}+a}{p}\right)\right|^{2}=17 p^{4}+4 p^{3} \alpha^{2}-88 p^{2} \alpha^{2}-116 p^{3}-17 p^{2}+120 p^{2} \alpha-4 p \alpha^{2}
$$

Notes: in Theorems 1 and 2, we only considered the twoterm exponential sums:

$$
S(m, k, p)=\sum_{a=1}^{p-1} e\left(\frac{m a^{k}+a}{p}\right)
$$

with $k=4$. Maybe we can use a similar method to study the general case $k \geq 3$, but it is hard to get an exact value for the hybrid power mean as in $H_{k}(p)$, except $k=4$. Therefore, we will not discuss the general integer $k$ in this paper.

Papers $[10,11]$ and our results are independent of each other. That is, they cannot be deduced from each other.

Even if our corollaries are used, they cannot be deduced with the results in [10].

In addition, our methods are not suitable for studying the mean value, 


$$
\sum_{m=1}^{p-1}\left(\sum_{b=0}^{p-1} e\left(\frac{m b^{4}}{p}\right)\right)^{k} \cdot\left|\sum_{a=1}^{p-1} e\left(\frac{m a^{4}+a}{p}\right)\right|^{3}
$$

because we cannot deal with the cubic power mean $\left|\sum_{a=1}^{p-1} e\left(m a^{4}+a / p\right)\right|^{3}$.

Maybe we can use our methods to study the fourth power mean:

$$
\sum_{m=1}^{p-1}\left(\sum_{b=0}^{p-1} e\left(\frac{m b^{4}}{p}\right)\right)^{k} \cdot\left|\sum_{a=1}^{p-1} e\left(\frac{m a^{4}+a}{p}\right)\right|^{4} .
$$

This is definitely a challenge, which is the goal of our further study.

\section{Several Lemmas}

In this section, we give some lemmas which are necessary in the proof of our theorems. Hereinafter, we shall use some properties of the classical Gauss sums and quadratic residue $\bmod p$, and all of them can be found in $[1,13]$, so they will not be repeated here. First, we have the following.
Lemma 1. Let $p$ be an odd prime with $p \equiv 1 \bmod 4$. Then, for any four-order character $\psi$ modulo $p$, we have the identity

$$
\tau^{2}(\psi)+\tau^{2}(\bar{\psi})=2 \sqrt{p} \cdot \alpha
$$

where $\tau(\chi)=\sum_{a=1}^{p-1} \chi(a) e(a / p)$ denotes the classical Gauss sums, $\alpha=\alpha(p)=(1 / 2) \sum_{a=1}^{p-1}(a+\bar{a} / p)$, and $(* / p)=\chi_{2} d e-$ notes Legendre's symbol modulo $p$.

Proof. See Lemma 2.2 in [9].

Lemma 2. Let $p$ be an odd prime with $p \equiv 1 \bmod 4$, and $(* / p)=\chi_{2}$ denotes the Legendre symbol $\bmod p$. Then, for any integer $b$ with $(b, p)=1$, we have the identities

$$
A(b)=\sum_{a=0}^{p-1} e\left(\frac{b a^{4}}{p}\right)=\left(\frac{b}{p}\right) \sqrt{p}+\sum_{a=1}^{p-1}\left(\frac{a}{p}\right) e\left(\frac{b a^{2}}{p}\right) .
$$

If $p=8 k+5$, then we have

$$
\begin{aligned}
A^{2}(b)= & -p+2\left(\frac{b}{p}\right) \sqrt{p}\left(\sum_{a=1}^{p-1}\left(\frac{a}{p}\right) e\left(\frac{b a^{2}}{p}\right)\right)+\left(\frac{b}{p}\right) \sqrt{p} \sum_{a=1}^{p-1}\left(\frac{a+\bar{a}}{p}\right), \\
A^{3}(b)= & -5\left(\frac{b}{p}\right) p^{(3 / 2)}+p\left(\sum_{a=1}^{p-1}\left(\frac{a}{p}\right) e\left(\frac{b a^{2}}{p}\right)\right)+3 p\left(\sum_{a=1}^{p-1}\left(\frac{a+\bar{a}}{p}\right)\right) \\
& +\left(\frac{b}{p}\right) \sqrt{p}\left(\sum_{a=1}^{p-1}\left(\frac{a}{p}\right) e\left(\frac{b a^{2}}{p}\right)\right)\left(\sum_{a=1}^{p-1}\left(\frac{a+\bar{a}}{p}\right)\right), \\
A^{4}(b)= & -2 p A^{2}(b)+4 p A(b)\left(\sum_{a=1}^{p-1}\left(\frac{a+\bar{a}}{p}\right)\right)-9 p^{2}+p\left(\sum_{a=1}^{p-1}\left(\frac{a+\bar{a}}{p}\right)\right)^{2} .
\end{aligned}
$$

If $p=8 k+1$, then we have

$$
\begin{aligned}
A^{2}(b)= & 3 p+2\left(\frac{b}{p}\right) \sqrt{p}\left(\sum_{a=1}^{p-1}\left(\frac{a}{p}\right) e\left(\frac{b a^{2}}{p}\right)\right)+\left(\frac{b}{p}\right) \sqrt{p} \sum_{a=1}^{p-1}\left(\frac{a+\bar{a}}{p}\right), \\
A^{3}(b)= & 7\left(\frac{b}{p}\right) p^{(3 / 2)}+5 p\left(\sum_{a=1}^{p-1}\left(\frac{a}{p}\right) e\left(\frac{b a^{2}}{p}\right)\right)+3 p\left(\sum_{a=1}^{p-1}\left(\frac{a+\bar{a}}{p}\right)\right) \\
& +\left(\frac{b}{p}\right) \sqrt{p}\left(\sum_{a=1}^{p-1}\left(\frac{a}{p}\right) e\left(\frac{b a^{2}}{p}\right)\right)\left(\sum_{a=1}^{p-1}\left(\frac{a+\bar{a}}{p}\right)\right) \\
A^{4}(b)= & 6 p A^{2}(b)+4 p A(b)\left(\sum_{a=1}^{p-1}\left(\frac{a+\bar{a}}{p}\right)\right)-p^{2}+p\left(\sum_{a=1}^{p-1}\left(\frac{a+\bar{a}}{p}\right)\right)^{2} .
\end{aligned}
$$


Proof. See Lemmas 1 and 3 in [12].

Lemma 3. Let $p$ be an odd prime with $p \equiv 1 \bmod 4$. Then, we have

$$
\sum_{m=1}^{p-1}\left|\sum_{a=1}^{p-1} e\left(\frac{m a^{4}+a}{p}\right)\right|^{2}=p^{2}-4 p-1
$$

Proof. Since $p \equiv 1 \bmod 4$, the congruence equation $x^{4} \equiv 1 \bmod p$ has four solutions. From the properties of reduced residue system $\bmod p$ and the trigonometric identity,

$$
\sum_{m=1}^{p-1} e\left(\frac{n m}{p}\right)= \begin{cases}p-1, & \text { if } p \mid n \\ -1, & \text { if } p+n\end{cases}
$$

we have

$$
\begin{aligned}
\sum_{m=1}^{p-1}\left|\sum_{a=1}^{p-1} e\left(\frac{m a^{4}+a}{p}\right)\right|^{2} & =\sum_{a=1}^{p-1} \sum_{b=1}^{p-1} \sum_{m=1}^{p-1} e\left(\frac{m b^{4}\left(a^{4}-1\right)+b(a-1)}{p}\right) \\
& =\sum_{a=1}^{p-1}\left(\sum_{m=1}^{p-1} e\left(\frac{m\left(a^{4}-1\right)}{p}\right)\right)\left(\sum_{b=1}^{p-1} e\left(\frac{b(a-1)}{p}\right)\right)=(p-1)^{2}-3(p-1)+(p-5)=p^{2}-4 p-1
\end{aligned}
$$

This proves Lemma 3.

$$
\sum_{m=1}^{p-1} \psi(m)\left|\sum_{a=1}^{p-1} e\left(\frac{m a^{4}+a}{p}\right)\right|^{2}=0 .
$$

Lemma 4. Let $p$ be an odd prime with $p \equiv 1 \bmod 4$. Then, for any four-order character $\psi \bmod p$ if $p=8 k+1$, we have the identity

$$
\sum_{m=1}^{p-1} \psi(m)\left|\sum_{a=1}^{p-1} e\left(\frac{m a^{4}+a}{p}\right)\right|^{2}=2 \tau(\psi)-2 \sqrt{p} \cdot \tau(\bar{\psi}) .
$$

Proof. For any integer $m$ with $(m, p)=1$, note that $1+$ $\psi(m)+\psi^{2}(m)+\bar{\psi}(m)=4$ if $m^{4} \equiv 1 \bmod p ; 1+\psi(m)$ $+\psi^{2}(m)+\bar{\psi}(m)=0$, otherwise. From (21) and the properties of Gauss sums, we have

If $p=8 k+5$, then we have

$$
\begin{aligned}
& \sum_{m=1}^{p-1} \psi(m)\left|\sum_{a=1}^{p-1} e\left(\frac{m a^{4}+a}{p}\right)\right|^{2}=\sum_{a=1}^{p-1} \sum_{b=1}^{p-1} \sum_{m=1}^{p-1} \psi(m) e\left(\frac{m b^{4}\left(a^{4}-1\right)+b(a-1)}{p}\right) \\
& =\tau(\psi) \sum_{a=1}^{p-1} \bar{\psi}\left(a^{4}-1\right) \sum_{b=1}^{p-1} e\left(\frac{b(a-1)}{p}\right)=-\tau(\psi) \sum_{a=1}^{p-1} \bar{\psi}\left(a^{4}-1\right) \\
& =-\tau(\psi) \sum_{a=1}^{p-1}\left(1+\psi(a)+\psi^{2}(a)+\bar{\psi}(a)\right) \bar{\psi}(a-1) \\
& =-\tau(\psi)\left(\sum_{a=1}^{p-1} \bar{\psi}(a-1)+\sum_{a=1}^{p-1} \bar{\psi}(1-\bar{a})\right)-\tau(\psi) \sum_{a=1}^{p-1} \chi_{2}(a) \bar{\psi}(a-1)-\tau(\psi) \sum_{a=1}^{p-1} \bar{\psi}(a(a-1)), \\
& \qquad \sum_{a=1}^{p-1} \bar{\psi}(a-1)+\sum_{a=1}^{p-1} \bar{\psi}(1-\bar{a})=2 \sum_{a=1}^{p-1} \bar{\psi}(a-1)=-2 .
\end{aligned}
$$

where we have used the identity $\chi_{2}=\psi^{2}$.

If $p=8 k+1$, then $\psi(-1)=1$ and 
From the properties of Gauss sums, $\tau\left(\chi_{2}\right)=\sqrt{p}$ and $\psi^{3}=\bar{\psi}$, we have

$$
\begin{aligned}
\sum_{a=1}^{p-1} \chi_{2}(a) \bar{\psi}(a-1) & =\frac{1}{\tau(\psi)} \sum_{b=1}^{p-1} \psi(b) \sum_{a=1}^{p-1} \chi_{2}(a) e\left(\frac{b(a-1)}{p}\right) \\
& =\frac{\sqrt{p}}{\tau(\psi)} \sum_{b=1}^{p-1} \psi(b) \chi_{2}(b) e\left(\frac{-b}{p}\right)=\sqrt{p} \cdot \frac{\tau(\bar{\psi})}{\tau(\psi)}, \\
\sum_{a=1}^{p-1} \bar{\psi}(a(a-1)) & =\frac{1}{\tau(\psi)} \sum_{b=1}^{p-1} \psi(b) \sum_{a=1}^{p-1} \bar{\psi}(a) e\left(\frac{b(a-1)}{p}\right) \\
& =\frac{\tau(\bar{\psi})}{\tau(\psi)} \sum_{b=1}^{p-1} \psi^{2}(b) e\left(\frac{-b}{p}\right)=\sqrt{p} \cdot \frac{\tau(\bar{\psi})}{\tau(\psi)} .
\end{aligned}
$$

If $p=8 k+1$, then combining (25)-(28), we have

$$
\sum_{m=1}^{p-1} \psi(m)\left|\sum_{a=1}^{p-1} e\left(\frac{m a^{4}+a}{p}\right)\right|^{2}=2 \tau(\psi)-2 \sqrt{p} \cdot \tau(\bar{\psi}) .
$$$$
\text { If } p=8 k+5 \text {, then } \psi(-1)=-1 \text {. Thus, }
$$

$$
\sum_{a=1}^{p-1} \bar{\psi}(a-1)+\sum_{a=1}^{p-1} \bar{\psi}(1-\bar{a})=\sum_{a=1}^{p-1} \bar{\psi}(a-1)+\sum_{a=1}^{p-1} \bar{\psi}(1-a)=0,
$$

$$
\sum_{a=1}^{p-1} \chi_{2}(a) \bar{\psi}(a-1)=\frac{\sqrt{p}}{\tau(\psi)} \sum_{b=1}^{p-1} \psi(b) \chi_{2}(b) e\left(\frac{-b}{p}\right)=-\sqrt{p} \cdot \frac{\tau(\bar{\psi})}{\tau(\psi)},
$$

$$
\sum_{a=1}^{p-1} \bar{\psi}(a(a-1))=\frac{\tau(\bar{\psi})}{\tau(\psi)} \sum_{b=1}^{p-1} \psi^{2}(b) e\left(\frac{-b}{p}\right)=\sqrt{p} \cdot \frac{\tau(\bar{\psi})}{\tau(\psi)} .
$$

Combining (25) and (30)-(32), we know that if $p=8 k+5$, then

$$
\sum_{m=1}^{p-1} \psi(m)\left|\sum_{a=1}^{p-1} e\left(\frac{m a^{4}+a}{p}\right)\right|^{2}=0 .
$$

Now, Lemma 4 follows from (26) and (33).

Lemma 5. Let $p$ be an odd prime with $p \equiv 1 \bmod 4$; then, we have

$$
\sum_{m=1}^{p-1} \chi_{2}(m)\left|\sum_{a=1}^{p-1} e\left(\frac{m a^{4}+a}{p}\right)\right|^{2}=2 \sqrt{p}-\psi(-1)\left(\tau^{2}(\psi)+\tau^{2}(\bar{\psi})\right) .
$$

Proof. Note that $\chi_{2}$ is a real character $\bmod p$ and $\tau\left(\chi_{2}\right)=\sqrt{p}$, and from the method of proving Lemma 3 , we have

$$
\begin{aligned}
& \sum_{m=1}^{p-1} \chi_{2}(m)\left|\sum_{a=1}^{p-1} e\left(\frac{m a^{4}+a}{p}\right)\right|^{2}=\sum_{a=1}^{p-1} \sum_{b=1}^{p-1} \sum_{m=1}^{p-1} \chi_{2}(m) e\left(\frac{m b^{4}\left(a^{4}-1\right)+b(a-1)}{p}\right) \\
& =\sqrt{p} \cdot \sum_{a=1}^{p-1} \chi_{2}\left(a^{4}-1\right) \sum_{b=1}^{p-1} e\left(\frac{b(a-1)}{p}\right)=-\sqrt{p} \cdot \sum_{a=1}^{p-1} \chi_{2}\left(a^{4}-1\right) \\
& =-\sqrt{p} \cdot \sum_{a=1}^{p-1}\left(1+\psi(a)+\chi_{2}(a)+\bar{\psi}(a)\right) \chi_{2}(a-1) \\
& =-\sqrt{p} \cdot\left(-2+\sum_{a=1}^{p-1} \psi(a) \chi_{2}(a-1)+\sum_{a=1}^{p-1} \bar{\psi}(a) \chi_{2}(a-1)\right)=2 \sqrt{p}-\psi(-1)\left(\tau^{2}(\psi)+\tau^{2}(\bar{\psi})\right) . \\
& \text { Lemma 5. } \quad \quad A(m)=\sum_{a=0}^{p-1} e\left(\frac{m a^{4}}{p}\right)=1+\sum_{a=1}^{p-1}\left(1+\psi(a)+\chi_{2}(a)+\bar{\psi}(a)\right) e\left(\frac{m a}{p}\right) \\
& \text { he Theorems } \quad=\sum_{a=0}^{p-1} e\left(\frac{m a}{p}\right)+\bar{\psi}(m) \tau(\psi)+\chi_{2}(m) \sqrt{p}+\psi(m) \tau(\bar{\psi}) \\
& \text { Thall complete the proofs of our theorems. } \\
& \text { Theorem 1. For any integer } m \text { with } \quad=\bar{\psi}(m) \tau(\psi)+\chi_{2}(m) \sqrt{p}+\psi(m) \tau(\bar{\psi}) .
\end{aligned}
$$

This proves Lemma 5.

\section{Proofs of the Theorems}

In this section, we shall complete the proofs of our theorems. First, we prove Theorem 1. For any integer $m$ with $(m, p)=1$, note that $\psi^{2}=\chi_{2}$ and $\tau\left(\chi_{2}\right)=\sqrt{p}$, and we have 
If $p$ is an odd prime with $p \equiv 1 \bmod 8$, then, applying (36) and Lemmas 1, 4, and 5 and noting that $\tau(\psi) \tau(\bar{\psi})=p$, we have

$$
\begin{aligned}
H_{1}(p) & =\sum_{m=1}^{p-1}\left(\bar{\psi}(m) \tau(\psi)+\chi_{2}(m) \sqrt{p}+\psi(m) \tau(\bar{\psi})\right)\left|\sum_{a=1}^{p-1} e\left(\frac{m a^{4}+a}{p}\right)\right|^{2} \\
& =\tau(\psi)(2 \tau(\bar{\psi})-2 \sqrt{p} \tau(\psi))+\tau(\bar{\psi})(2 \tau(\psi)-2 \sqrt{p} \tau(\bar{\psi}))+\sqrt{p}\left(2 \sqrt{p}-\tau^{2}(\psi)-\tau^{2}(\bar{\psi})\right) \\
& =6 p-3 \sqrt{p} \cdot\left(\tau^{2}(\psi)+\tau^{2}(\bar{\psi})\right)=6 p \cdot(1-\alpha) .
\end{aligned}
$$

Similarly, note that the identity

$\tau^{4}(\psi)+\tau^{4}(\bar{\psi})=\left(\tau^{2}(\psi)+\tau^{2}(\bar{\psi})\right)^{2}-2 p^{2}=2 p \cdot\left(2 \alpha^{2}-p\right)$.
From the method of proving (37) and Lemmas 1 and 2, we also have

$$
\begin{aligned}
& H_{2}(p)=\sum_{m=1}^{p-1}\left(\bar{\psi}(m) \tau(\psi)+\chi_{2}(m) \sqrt{p}+\psi(m) \tau(\bar{\psi})\right)^{2}\left|\sum_{a=1}^{p-1} e\left(\frac{m a^{4}+a}{p}\right)\right|^{2} \\
& =\sum_{m=1}^{p-1}\left(3 p+\chi_{2}(m) \tau^{2}(\psi)+\chi_{2}(m) \tau^{2}(\bar{\psi})+2 \sqrt{p} \psi(m) \tau(\psi)+2 \sqrt{p} \bar{\psi}(m) \tau(\bar{\psi})\right) \times\left|\sum_{a=1}^{p-1} e\left(\frac{m a^{4}+a}{p}\right)\right|^{2} \\
& =3 p\left(p^{2}-4 p-1\right)+\left(\tau^{2}(\psi)+\tau^{2}(\bar{\psi})\right)\left(2 \sqrt{p}-\tau^{2}(\psi)-\tau^{2}(\bar{\psi})\right)+2 \sqrt{p} \tau(\psi) \\
& (2 \tau(\psi)-2 \sqrt{p} \tau(\bar{\psi}))+2 \sqrt{p} \tau(\bar{\psi})(2 \tau(\bar{\psi})-2 \sqrt{p} \tau(\psi)) \\
& =3 p^{3}-22 p^{2}-3 p+6 \sqrt{p}\left(\tau^{2}(\psi)+\tau^{2}(\bar{\psi})\right)-\left(\tau^{4}(\psi)+\tau^{4}(\bar{\psi})\right)=3 p^{3}-20 p^{2}-4 p \alpha^{2}+12 p \alpha-3 p, \\
& H_{3}(p)=\sum_{m=1}^{p-1}\left(\bar{\psi}(m) \tau(\psi)+\chi_{2}(m) \sqrt{p}+\psi(m) \tau(\bar{\psi})\right)^{3}\left|\sum_{a=1}^{p-1} e\left(\frac{m a^{4}+a}{p}\right)\right|^{2} \\
& =\sum_{m=1}^{p-1}\left(\begin{array}{c}
\psi(m) \tau^{3}(\psi)+\bar{\psi}(m) \tau^{3}(\bar{\psi})+\chi_{2}(m) p \sqrt{p}+3 p \bar{\psi}(m) \tau(\psi)+3 p \psi(m) \tau(\bar{\psi}) \\
+3 \sqrt{p} \tau^{2}(\psi)+3 \sqrt{p} \tau^{2}(\bar{\psi})+3 p \bar{\psi}(m) \tau(\psi)+3 p \psi(m) \tau(\bar{\psi})+6 p^{(3 / 2)} \chi_{2}(m)
\end{array}\right) \times\left|\sum_{a=1}^{p-1} e\left(\frac{m a^{4}+a}{p}\right)\right|^{2} \\
& =\tau^{3}(\psi)(2 \tau(\psi)-2 \sqrt{p} \tau(\bar{\psi}))+\tau^{3}(\bar{\psi})(2 \tau(\bar{\psi})-2 \sqrt{p} \tau(\psi))+p^{(3 / 2)}\left(2 \sqrt{p}-\tau^{2}(\psi)-\tau^{2}(\bar{\psi})\right) \\
& +3 \sqrt{p}\left(p^{2}-4 p-1\right)\left(\tau^{2}(\psi)+\tau^{2}(\bar{\psi})\right)+6 p \tau(\psi)(2 \tau(\bar{\psi})-2 \sqrt{p} \tau(\psi))+6 p \tau(\bar{\psi})(2 \tau(\psi)-2 \sqrt{p} \cdot \tau(\bar{\psi})) \\
& +6 p^{(3 / 2)}\left(2 \sqrt{p}-\tau^{2}(\psi)-\tau^{2}(\bar{\psi})\right)=3 p^{(1 / 2)}\left(p^{2}-11 p-1\right)\left(\tau^{2}(\psi)+\tau^{2}(\bar{\psi})\right)+2\left(\tau^{4}(\psi)+\tau^{4}(\bar{\psi})\right)+38 p^{2} \\
& =6 p^{3} \alpha-66 p^{2} \alpha-6 p \alpha+8 p \alpha^{2}+34 p^{2} \text {, }
\end{aligned}
$$




$$
\begin{aligned}
H_{4}(p)= & \sum_{m=1}^{p-1}\left(\bar{\psi}(m) \tau(\psi)+\chi_{2}(m) \sqrt{p}+\psi(m) \tau(\bar{\psi})\right)^{4}\left|\sum_{a=1}^{p-1} e\left(\frac{m a^{4}+a}{p}\right)\right|^{2} \\
= & \sum_{m=1}^{p-1}\left(\begin{array}{c}
\tau^{4}(\psi)+\tau^{4}(\bar{\psi})+p^{2}+6 p^{2}+6 p \chi_{2}(m) \tau^{2}(\psi)+6 p \chi_{2}(m) \tau^{2}(\bar{\psi}) \\
+12 p^{2}+12 p^{(3 / 2)} \psi(m) \tau(\psi)+12 p^{(3 / 2)} \bar{\psi}(m) \tau(\bar{\psi})+4 \sqrt{p} \bar{\psi}(m) \tau^{3}(\psi)+ \\
4 \sqrt{p} \psi(m) \tau^{3}(\bar{\psi})+4 p \chi_{2}(m) \tau^{2}(\psi)+4 p \chi_{2}(m) \tau^{2}(\bar{\psi})+4 p^{(3 / 2)} \psi(m) \tau(\psi) \\
\quad+4 p^{(3 / 2)} \bar{\psi}(m) \tau(\bar{\psi})
\end{array}\right)\left|\sum_{a=1}^{p-1} e\left(\frac{m a^{4}+a}{p}\right)\right|^{2} \\
= & \left(\tau^{4}(\psi)+\tau^{4}(\bar{\psi})+19 p^{2}\right)\left(p^{2}-4 p-1\right)+10 p\left(\tau^{2}(\psi)+\tau^{2}(\bar{\psi})\right)\left(2 \sqrt{p}-\tau^{2}(\psi)-\tau^{2}(\bar{\psi})\right) \\
& +16 p^{(3 / 2)} \tau(\psi)(2 \tau(\psi)-2 \sqrt{p} \cdot \tau(\bar{\psi}))+16 p^{(3 / 2)} \tau(\bar{\psi})(2 \tau(\bar{\psi})-2 \sqrt{p} \cdot \tau(\psi))+4 \sqrt{p} \tau^{3}(\bar{\psi}) \\
( & 2 \tau(\psi)-2 \sqrt{p} \cdot \tau(\bar{\psi}))+4 \sqrt{p} \tau^{3}(\psi)(2 \tau(\bar{\psi})-2 \sqrt{p} \cdot \tau(\psi)) \\
= & \left(\tau^{4}(\psi)+\tau^{4}(\bar{\psi})\right)\left(p^{2}-22 p-1\right)+60 p^{(3 / 2)}\left(\tau^{2}(\psi)+\tau^{2}(\bar{\psi})\right)+p^{2}\left(19 p^{2}-160 p-19\right) \\
= & 17 p^{4}+4 p^{3} \alpha^{2}-88 p^{2} \alpha^{2}-116 p^{3}-17 p^{2}+120 p^{2} \alpha-4 p \alpha^{2} .
\end{aligned}
$$

If $k \geq 1$, then, from Lemmas 1 and 2, we have

$$
\begin{aligned}
H_{k+4}(p) & =\sum_{m=1}^{p-1} A^{k}(m) \cdot A^{4}(m) \cdot\left|\sum_{a=1}^{p-1} e\left(\frac{m a^{4}+a}{p}\right)\right|^{2} \\
& =\sum_{m=1}^{p-1} A^{k}(m) \cdot\left(6 p A^{2}(m)+8 p \alpha A(m)-\left(p^{2}-4 p \alpha^{2}\right)\right) \cdot\left|\sum_{a=1}^{p-1} e\left(\frac{m a^{4}+a}{p}\right)\right|^{2} \\
& =6 p H_{k+2}(p)+8 p \alpha H_{k+1}(p)-\left(p^{2}-4 p \alpha^{2}\right) H_{k}(p) .
\end{aligned}
$$

Now, Theorem 1 follows from (37)-(42).

Proof of Theorem 2. If the prime $p$ satisfies $p=8 k+5$, then note that $\psi(-1)=-1$, and from (13) and Lemmas $1,3,4$, and 5 , we have

$$
\begin{aligned}
H_{1}(p) & =\sum_{m=1}^{p-1}\left(\bar{\psi}(m) \tau(\psi)+\chi_{2}(m) \sqrt{p}+\psi(m) \tau(\bar{\psi})\right)\left|\sum_{a=1}^{p-1} e\left(\frac{m a^{4}+a}{p}\right)\right|^{2} \\
& =\sqrt{p}\left(2 \sqrt{p}+\tau^{2}(\psi)+\tau^{2}(\bar{\psi})\right)=2 p \cdot(\alpha+1) .
\end{aligned}
$$

Similarly, note that $\tau(\psi) \tau(\bar{\psi})=-p$; from Lemmas $1-5$, we also have 


$$
\begin{aligned}
& H_{2}(p)=\sum_{m=1}^{p-1}\left(\bar{\psi}(m) \tau(\psi)+\chi_{2}(m) \sqrt{p}+\psi(m) \tau(\bar{\psi})\right)^{2}\left|\sum_{a=1}^{p-1} e\left(\frac{m a^{4}+a}{p}\right)\right|^{2} \\
& =\sum_{m=1}^{p-1}\left(-p+\chi_{2}(m) \tau^{2}(\psi)+\chi_{2}(m) \tau^{2}(\bar{\psi})+2 \sqrt{p} \psi(m) \tau(\psi)+2 \sqrt{p} \bar{\psi}(m) \tau(\bar{\psi})\right) \times\left|\sum_{a=1}^{p-1} e\left(\frac{m a^{4}+a}{p}\right)\right|^{2} \\
& =-p\left(p^{2}-4 p-1\right)+\left(\tau^{2}(\psi)+\tau^{2}(\bar{\psi})\right)\left(2 \sqrt{p}+\tau^{2}(\psi)+\tau^{2}(\bar{\psi})\right)=-p^{3}+4 p^{2}+p+4 p \alpha^{2}+4 p \alpha, \\
& H_{3}(p)=\sum_{m=1}^{p-1}\left(\bar{\psi}(m) \tau(\psi)+\chi_{2}(m) \sqrt{p}+\psi(m) \tau(\bar{\psi})\right)^{3}\left|\sum_{a=1}^{p-1} e\left(\frac{m a^{4}+a}{p}\right)\right|^{2} \\
& =\sum_{m=1}^{p-1}\left(\begin{array}{c}
\psi(m) \tau^{3}(\psi)+\bar{\psi}(m) \tau^{3}(\bar{\psi})+\chi_{2}(m) p \sqrt{p}-3 p \bar{\psi}(m) \tau(\psi)-3 p \psi(m) \tau(\bar{\psi}) \\
+3 \sqrt{p} \tau^{2}(\psi)+3 \sqrt{p} \tau^{2}(\bar{\psi})+3 p \bar{\psi}(m) \tau(\psi)+3 p \psi(m) \tau(\bar{\psi})-6 p^{(3 / 2)} \chi_{2}(m)
\end{array}\right) \times\left|\sum_{a=1}^{p-1} e\left(\frac{m a^{4}+a}{p}\right)\right|^{2} \\
& =-5 p^{(3 / 2)}(2 \sqrt{p}+G(2, \psi))+3 \sqrt{p} G(2, \psi)\left(p^{2}-4 p-1\right)=-10 p^{2}+2 \alpha \cdot\left(3 p^{3}-17 p^{2}-3 p\right) \text {, } \\
& H_{4}(p)=\sum_{m=1}^{p-1}\left(\bar{\psi}(m) \tau(\psi)+\chi_{2}(m) \sqrt{p}+\psi(m) \tau(\bar{\psi})\right)^{4}\left|\sum_{a=1}^{p-1} e\left(\frac{m a^{4}+a}{p}\right)\right|^{2} \\
& =\sum_{m=1}^{p-1}\left(\begin{array}{c}
\tau^{4}(\psi)+\tau^{4}(\bar{\psi})+p^{2}+6 p^{2}+6 p \chi_{2}(m) \tau^{2}(\psi)+6 p \chi_{2}(m) \tau^{2}(\bar{\psi}) \\
-12 p^{2}-12 p^{(3 / 2)} \psi(m) \tau(\psi)-12 p^{(3 / 2)} \bar{\psi}(m) \tau(\bar{\psi})+4 \sqrt{p} \bar{\psi}(m) \tau^{3}(\psi) \\
+4 \sqrt{p} \psi(m) \tau^{3}(\bar{\psi})-4 p \chi_{2}(m) \tau^{2}(\psi)-4 p \chi_{2}(m) \tau^{2}(\bar{\psi}) \\
+4 p^{(3 / 2)} \psi(m) \tau(\psi)+4 p^{(3 / 2)} \bar{\psi}(m) \tau(\bar{\psi})
\end{array}\right)\left|\sum_{a=1} e\left(\frac{m a^{4}+a}{p}\right)\right|^{2} \\
& =\left(\tau^{4}(\psi)+\tau^{4}(\bar{\psi})-5 p^{2}\right)\left(p^{2}-4 p-1\right)+2 p\left(\tau^{2}(\psi)+\tau^{2}(\bar{\psi})\right)\left(2 \sqrt{p}+\tau^{2}(\psi)+\tau^{2}(\bar{\psi})\right) \\
& =G(4, \psi)\left(p^{2}-2 p-1\right)+4 p^{(3 / 2)} G(2, \psi)-p^{2}\left(5 p^{2}-24 p-5\right) \\
& =4 p^{3} \alpha^{2}-7 p^{4}-8 p^{2} \alpha^{2}+28 p^{3}-4 p \alpha^{2}+7 p^{2}+8 p^{2} \alpha \text {. }
\end{aligned}
$$

If $k \geq 1$, then, from Lemmas 1 and 2 , we have

$$
\begin{aligned}
H_{k+4}(p) & =\sum_{m=1}^{p-1} A^{k}(m) \cdot A^{4}(m) \cdot\left|\sum_{a=1}^{p-1} e\left(\frac{m a^{4}+a}{p}\right)\right|^{2} \\
& =\sum_{m=1}^{p-1} A^{k}(m) \cdot\left(-2 p A^{2}(m)+8 p \alpha A(m)-\left(9 p^{2}-4 p \alpha^{2}\right)\right) \cdot\left|\sum_{a=1}^{p-1} e\left(\frac{m a^{4}+a}{p}\right)\right|^{2} \\
& =-2 p H_{k+2}(p)+8 p \alpha H_{k+1}(p)-\left(9 p^{2}-4 p \alpha^{2}\right) H_{k}(p) .
\end{aligned}
$$

Now, Theorem 2 follows from (43)-(47).

This completes the proofs of our all theorems.

\section{Conclusion}

The main results of this paper are two theorems. Theorem 1 establishes a four-order linear recurrence formula for the hybrid power mean involving the quartic Gauss sums and the two-term exponential sums for the case $p \equiv 1 \bmod 8$. Theorem 2 establishes a similar conclusion for the case $p \equiv 5 \bmod 8$. These achievements represent new contributions to research in the relevant fields, and it also has a good reference function to the research of related problems.

\section{Data Availability}

No data were used to support the findings of the study.

\section{Conflicts of Interest}

The authors declare that there are no conflicts of interest regarding the publication of this paper. 


\section{Authors' Contributions}

All authors have equally contributed to this work and have read and approved the final manuscript.

\section{Acknowledgments}

This work was supported by the N. S .F. (11771351) of P. R. China.

\section{References}

[1] W. P. Zhang and H. L. Li, Elementary Number Theory, Shaanxi Normal University Press, Xi'an, China, 2008.

[2] J. Bourgain, M. Z. Garaev, S. V. Konyagin, and I. E. Shparlinski, "On the hidden shifted power problem," Siam Journal on Computing, vol. 41, no. 6, pp. 1524-1557, 2012.

[3] D. A. Burgess, "On Dirichlet characters of polynomials," Proceedings of the London Mathematical Society, vol. 13, no. 1, pp. 537-548, 1963.

[4] A. Granville and K. Soundararajan, "Large character sums: pretentious characters and the Plya-Vinogradov theorem," Journal of the American Mathematical Society, vol. 20, pp. 357-384, 2007.

[5] A. Weil, "On some exponential sums," Poceedings of the National Academy of Sciences of the United States of America, vol. 34, no. 5, pp. 204-207, 1948.

[6] W. P. Zhang and H. N. Liu, "On the general Gauss sums and their fourth power mean," Osaka Journal of Mathematics, vol. 42, pp. 189-199, 2005.

[7] W. Zhang and W. Yao, "A note on the Dirichlet characters of polynomials,” Acta Arithmetica, vol. 115, no. 3, pp. 225-229, 2004.

[8] W. P. Zhang and Y. Yi, "On Dirichlet characters of polynomials," Bulletin of the London Mathematical Society, vol. 34, pp. 469-473, 2002.

[9] Z. Y. Chen and W. P. Zhang, "On the fourth-order linear recurrence formula related to classical Gauss sums," Open Mathematics, vol. 15, pp. 1251-1255, 2017.

[10] X. X. Li, "The hybrid power mean of the quartic Gauss sums and the two-term exponential sums," Advances in Difference Equations, vol. 2018, Article ID 236, 2018.

[11] S. Shen, "On the recursive properties of one kind hybrid power mean involving two-term exponential sums and Gauss sums," Open Mathematics, vol. 16, no. 1, pp. 955-966, 2018.

[12] S. M. Shen and W. P. Zhang, "On the quartic Gauss sums and their recurrence property," Advances in Difference Equations, vol. 2017, p. 43, 2017.

[13] T. M. Apostol, Introduction to Analytic Number Theory, Springer-Verlag, Berlin, Germany, 1976. 